\title{
A Fuzzy Logic-Possibilistic Methodology to Analyze the Main Corrosion Damages Mechanisms in Pipes and Equipment Installed in an Oil and Gas Platform
}

\author{
Giraldes Luis Nathan Leal ${ }^{*}$, Haddad Assed Naked \\ Environmental Engineering Program, Federal University of Rio de Janeiro, Rio de Janeiro, Brazil \\ Email address: \\ nathangiraldes@poli.ufrj.br(G. L. N. Leal), assed@poli.ufrj.br(H. A. Naked) \\ ${ }^{*}$ Corresponding author \\ To cite this article: \\ Giraldes Luis Nathan Leal, Haddad Assed Naked. A Fuzzy Logic-Possibilistic Methodology to Analyze the Main Corrosion Damages \\ Mechanisms in Pipes and Equipment Installed in an Oil and Gas Platform. International Journal of Science and Qualitative Analysis. \\ Vol. 5, No. 1, 2019, pp. 15-23. doi: 10.11648/j.ijsqa.20190501.13
}

Received: April 20, 2019; Accepted: June 5, 2019; Published: June 20, 2019

\begin{abstract}
Industrial accidents in recent years, particularly in the 1980s, have contributed significantly to the attention of government authorities, industry and society as a whole, in order to seek mechanisms to prevent such episodes that compromise safety of people and the quality of the environment. Currently one of the most discussed topics in various industries is process safety. Not all hazards and risks are the same or can have the same consequences. Process hazards and risks can cause major accidents, involving the release of potentially hazardous materials, fires, and explosions, or both. Accident studies have shown that equipment malfunctions are one of the major causes of unexpected and undesirable events, and so the inspection has been a technique to examine the actual condition of equipment exposed to corrosion damage mechanisms. One of the outputs from the inspection process is the observation of which damage mechanism is acting more intensely on the equipment or the piping. Having this information can help in forecasting the corrosion rates, which consequently assists in the design of a better inspection and maintenance plan. This work presents a methodology based on the Fuzzy logic, to analyze which are the corrosion damages mechanisms that contribute most to the deterioration of the equipment and pipes in an oil platform.
\end{abstract}

Keywords: Process Safety, Inspection, Corrosion, Damage Mechanisms, Fuzzy Logic

\section{Introduction}

After World War II, the demand for new materials intensifies and according to the International Disaster Database (EM-DAT), there have never been as many records of technological accidents as in the last decades (Figure. 1). This ends up triggering a change in the importance of "Safety", which has become an important factor to be considered due to the increase of the complexity of processes (operations with high temperatures and pressures) in the industries that can cause technological accidents, and with this, itself has been transformed, leaving aside the emphasis only on personal safety and on what we might call loss prevention. The operation of the process is the most dangerous activity alongside the transportation and drilling operation on an offshore platform [1].
Past experiences of onshore and offshore oil and gas activities have revealed that a small error in the operation of the process can turn into a catastrophe. This is a special concern in the platform due to the limited space and compact geometry of the process area, less ventilation, and difficult escape routes. On a platform, each extra control measure, which is implemented, not only occupies platform space and increases congestion, but also adds extra load to the platform. The offshore oil industry has developed rapidly in recent years. Despite technical development, several accidents have occurred over the years with severe environmental consequences and multiple worker fatalities. 


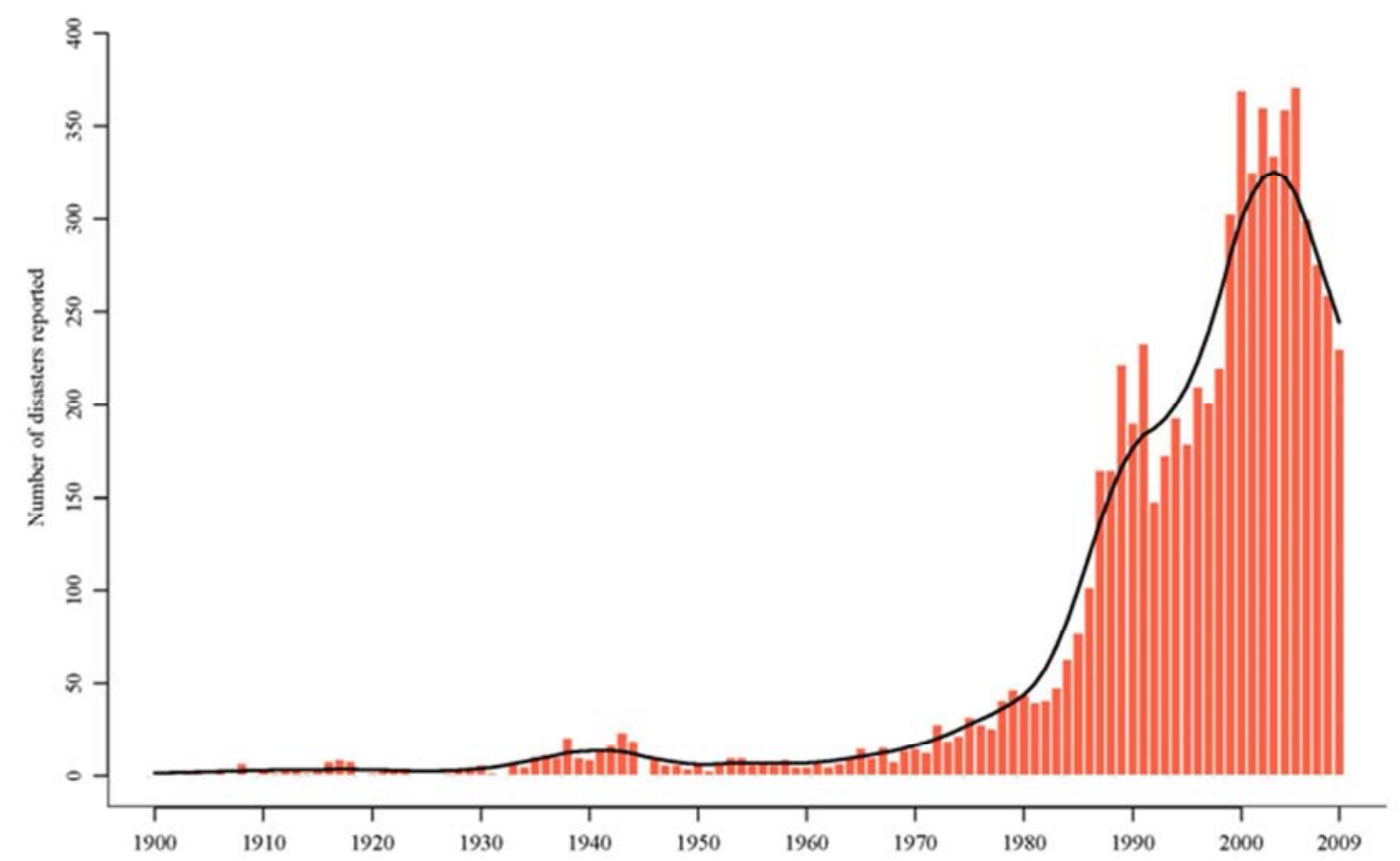

Figure 1. Technological accidents (EM-DAT: The OFDA/CRED Internationl Disaster Database - www.emdat.be - Université Catholique de Loughin, Brussels - Belgium).

Usually, there are large inventories of flammable and polluting products which implies great potential "destroyers". In addition, the offshore production units are congested and with numerous points of possible leaks. For all these characteristics, the offshore oil industry is required to do thorough risk management. And one of the primary goals of this risk management is to make the likelihood of a containment loss occur by avoiding leaks that could result in fire and explosion. Many variables should be part of the equation that seeks the best results in terms of reducing the likelihood of loss of contention, but certainly, knowing the mechanisms of damages that act on equipment and pipelines will help to act in a preventive way before these mechanisms end with the integrity of the material.

\section{Damage Mechanisms}

According to the API Recommended Practice 581, that provides guidance on developing a risk-based inspection (RBI) program for fixed equipment and pipe in the hydrocarbon and chemical process industries; understanding equipment operation and the interaction with the process environment (both internal and external) and the mechanical environment is key to identifying damage mechanisms. The equipment must have its inspection plans developed according to the mechanisms of damages expected for the type of service to which they will be submitted. Appropriate inspection techniques should be selected for each type of damage mechanism. In general, the damages and deteriorations that occur most frequently in static pressure equipment in industrial units are: a. Corrosion;

b. Loss of thickness;

c. Metallurgical changes;

d. Flaws;

e. Dimensional changes;

f. Puffing;

g. -Fouling.

It is verified that among the several mechanisms of deterioration presented, the corrosive processes deserve highlights, as they often cause serious damages to the process equipment.

\section{Corrosive Processes}

According to Telles (2003), corrosion is a set of phenomena of progressive deterioration of materials, mainly metallic, because of chemical or electrochemical reactions between the material and the environmental.

According to Gemelli (2001), corrosion is an interface phenomenon that affects organic materials and mainly inorganic materials, being manifested through chemical or electrochemical reactions. In the first case, the electron transfer takes place directly between the two chemical species involved. Already in electrochemical corrosion, the electrons are transferred indirectly, that is, they diffuse through the surface of the solid (metal or film) to a point where they are received by the element of the medium (oxidant). In this case, the receptor and the electron donor are in different places, in opposition to the chemical reactions, where the donor and the receiver are located at the same point of the surface of the material. 


\begin{tabular}{|c|c|c|c|}
\hline DM\# & Damage Mechanism & $\mathrm{DM}=$ & Damage Mechanism \\
\hline 1 & Sulfidation & 34 & Softening (Spheroidization) \\
\hline 2 & $\begin{array}{l}\text { Wet } \mathrm{H}_{2} \mathrm{~S} \text { Damage } \\
\text { (Blistering/HIC/SOHICISSC) }\end{array}$ & 35 & Reheat Cracking \\
\hline 3 & Creep / Stress Rupture & 36 & Sulfuric Acid Corrosion \\
\hline 4 & High temp $\mathrm{H}_{2} / \mathrm{H}_{6} \mathrm{~S}$ Corrosion & 37 & Hydrofluoric Acid Corrosion \\
\hline 5 & Polythionic Acid Cracking & 38 & Flue Gas Dew Point Corrosion \\
\hline 6 & Naphthenic Acid Corrosion & 39 & Dissimilar Metal Weld (DMW) Cracking \\
\hline 7 & Ammonium Bisulfide Corrosion & 40 & Hydrogen Stress Cracking in HF \\
\hline 8 & Ammonium Chloride Corrosion & 41 & Dealloying (Dezincification/ Denickelification) \\
\hline 9 & $\mathrm{HCl}$ Corrosion & 42 & $\mathrm{CO}_{2}$ Corrosion \\
\hline 10 & High Temperature Hydrogen Attack & 43 & Corrosion Fatigue \\
\hline 11 & Oxidation & 44 & Fuel Ash Corrosion \\
\hline 12 & Thermal Fatigue & 45 & Amine Corrosion \\
\hline 13 & Sour Water Corrosion (acidic) & 46 & Corrosion Under Insulation (CUI) \\
\hline 14 & Refractory Degradation & 47 & Atmospheric Corrosion \\
\hline 15 & Graphitization & 48 & Ammonia Stress Corrosion Cracking \\
\hline 16 & Temper Embrittlement & 49 & Cooling Water Corrosion \\
\hline 17 & Decarburization & 50 & Boiler Water / Condensate Corrosion \\
\hline 18 & Caustic Cracking & 51 & Microbiologically Induced Corrosion (MIC) \\
\hline 19 & Caustic Corrosion & 52 & Liquid Metal Embrittlement \\
\hline 20 & Erosion / Erosion-Corrosion & 53 & Galvanic Corrosion \\
\hline 21 & Carbonate SCC & 54 & Mechanical Fatigue \\
\hline 22 & Amine Cracking & 55 & \begin{tabular}{|l|} 
Nitriding \\
\end{tabular} \\
\hline 23 & Chloride Stress Corrosion Cracking & 56 & Vibration-Induced Fatigue \\
\hline 24 & Carburization & 57 & Titanium Hydriding \\
\hline 25 & Hydrogen Embrittlement & 58 & \begin{tabular}{|l|} 
Soil Corrosion \\
\end{tabular} \\
\hline 26 & Steam Blanketing & 59 & Metal Dusting \\
\hline 27 & Thermal Shock & 60 & Strain Aging \\
\hline 28 & Cavitation & 61 & Sulfate Stress Corrosion Cracking \\
\hline 29 & Graphitic Corrosion (see Dealloying) & 62 & Phosphoric Acid Corrosion \\
\hline 30 & Short term Overheating - Stress Rupture & 63 & Phenol (carbolic acid) Corrosion \\
\hline 31 & Brittle Fracture & 64 & Ethanol Stress Corrosion Cracking \\
\hline 32 & Sigma Phase/ Chi Embrittlement & 65 & Oxygen-Enhanced Ignition and Combustion \\
\hline 33 & $885^{\circ} \mathrm{F}\left(475^{\circ} \mathrm{C}\right)$ Embrittlement & 66 & $\begin{array}{l}\text { Organic Acid Corrosion Of Distillation Tower } \\
\text { Overhead Systems }\end{array}$ \\
\hline
\end{tabular}

Figure 2. List of the main Damage Mechanisms according to API 571 -Damage mechanisms affecting fixed equipment in the refining industry.

According to API 571, erosion-corrosion is a description for the damage that occurs when corrosion contributes to erosion by removing protective films or scales, or by exposing the metal surface to further corrosion under the combined action of erosion and corrosion $[2,16]$. The materials affected are all metals, alloys, and refractories, and still based on API 571, the main critical factors are:

a. Metal loss rate depends on the velocity and concentration of impacting medium (i.e., particles, liquids, droplets, slurries, two-phase flow), the size and hardness of impacting particles, the hardness and corrosion resistance of material subject to erosion, and the angle of impact.

b. Softer alloys such as copper and aluminium alloys that are easily worn from mechanical damage may be subject to severe metal loss under high-velocity conditions.

c. Although the increasing hardness of the metal substrate is a common approach to minimize damage, it is not always a good indicator of improved resistance to erosion, particularly where corrosion plays a significant role.

d. Increasing the corrosivity of the environment may reduce the stability of protective surface films and increase the susceptibility to metal loss. The metal may be removed from the surface as dissolved ions, or as solid corrosion products which are mechanically swept from the metal surface.

e. Factors which contribute to an increase in corrosivity of the environment, such as temperature, $\mathrm{pH}$, etc., can increase susceptibility to metal loss.

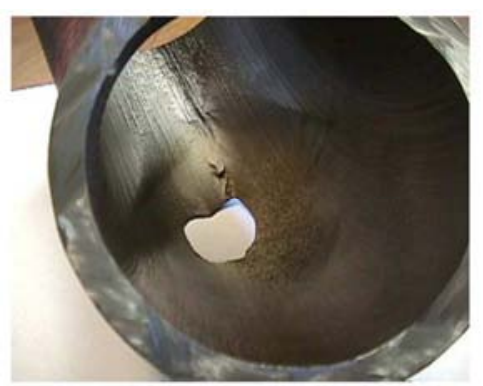

(a)

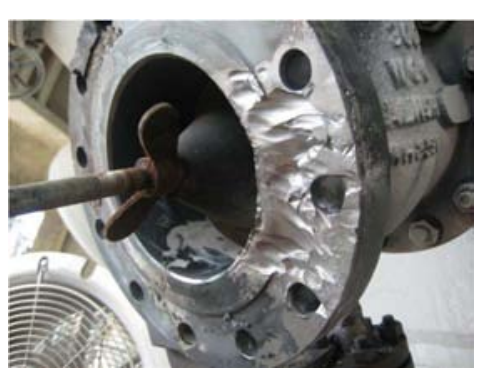

(b)

Figure 3. (a) Erosion of a 9Cr-1Mo coker heater return bend; (b) Erosion corrosion of a $1.25 \mathrm{Cr} 300$ \# Valve flange on an FCC Catalyst withdrawal line. (source: API 571). 


\section{Inspection Process in Platform of Petroleum}

As already mentioned, inspection is a very important part of the preventive process, to guarantee the equipment and pipes are working properly, into a good condition. The Risk Based Inspection (RBI) can be applied to pipes at all stages of their life $[3,12,13]$. Many petroleum companies are using a robust system called RBI - Risk Based Inspection- it is a program to optimize inspection strategy based on risk prioritization. The process consists of detail risk analysis; comprising probability of failure and consequence of failures, and actions to minimize risk exposure to as low as reasonably practicable (ALARP) by utilizing optimum, effective, efficient inspection programs, and proper mitigation activities. Our focus is a platform of petroleum, the RBI covers static pressurized equipment and piping on both topside and marine systems, such as piping, separators, scrubbers, drums, filters, columns, heat exchangers, etc. RBI program is a continuous process which requires the involvement of multiple ranges of disciplines. As a minimum, RBI team should consist of RBI Engineer, Integrity Engineer, Technical \& Maintenance Manager (TMM), Inspectors/Roving Team, and Offshore team which includes Operation Installation Manager (OIM) and Superintendents (Production, Maintenance, and Marine Superintendent) [4]. RBI Engineer shall be the focal point to lead RBI assessment and ensure that the RBI program meets requirements in this procedure and all key elements as per API RP 580 [15]. The output from RBI is a report describing all basis of assessment and process during conducting RBI assessment. The report should consist of RBI methodology, input data, POF (Probability of failure) determination, COF (Consequence of failure) determination, risk distribution, risk prioritization, inspection planning, and all assumptions which are used during the assessment, such as damage mechanisms.

\section{Fuzzy Methodology}

There are some phases of a process risk analysis, that the actual situation is often not sharp and deterministic due to the number of uncertainties. These uncertainties can be classified into two groups: as "objective uncertainties", which arise from a random character of the evaluation process (variability) and "subjective uncertainties", arising from limited and partial knowledge and information (imprecision). In such a situation, fuzzy logic can be used. According to Zadeh, fuzzy logic or fuzzy set theory can work with uncertainty and precision and can solve problems where there are no precise boundaries and precise values [5]. The concept of a fuzzy set provides mathematical formulations that can characterize the uncertain parameters involved in the method of risk analysis. According to CHAMOVITZ and COSENZA (2010), the use of fuzzy logic in will be indicated whenever one wishes to approach the constructed model of reality [6] [14]. This assertion is based on the principle of incompatibility established by ZADEH (1973): "As the complexity of a system increases, our ability to make accurate and meaningful statements about this system decreases until a threshold is reached beyond which accuracy and significance (or relevance) become almost mutually exclusive characteristics. In fuzzy logic, the equivalent of traditional independent variables, fuzzy sets are defined for specific linguistic variables. The selected categories of each variable constitute the fuzzy sets. A fuzzy set defined in a discourse universe $(U)$ is characterized by an association function $(\mathrm{X})$, which assumes values of the interval $[0,1]$. An association function provides a measure of the degree of similarity of a U-element to the diffuse subset.

The use of fuzzy logic in relation to classical logic in complex processes is justified according to JANG and GULLEY, 1995:

a. Because the naturalness of your approach makes your concepts easy to understand.

b. Because it is flexible. - Because it is tolerant of inaccurate data.

c. Because it can model nonlinear functions of arbitrariness complexity.

d. Because it can be built based on expert experience.

e. Because it is based on natural language, the basis of human communication

\subsection{Steps to Use the Fuzzy Logic}

As already explained, the Fuzzy logic (FL) has as an objective to act in subjective processes, making, through a series of specific analyzes of indicators, and mathematics resources of sets and matrices, an analysis is made that has as output numbers that generate more precise data and reliable [7].

Basically, the step-by-step (Figure 4) to make analyzes based on Fuzzy is:

a. Definition of indicators: which are exactly the data we want to analyze and seek greater accuracy.

b. Selection of specialists. The theory of fuzzy sets allows us to represent the knowledge elicited by means of pertinence functions. A fuzzy model built with the help of experts increases its accuracy as the number of specialists recognized by their knowledge, experience, and work in the area of interest increases.

c. Determination of the degree of importance of the specialists. Since a specialist group is usually heterogeneous, opinions can be considered with the same intensity, that is, with the same degree of importance. In this way, each opinion will have importance given the degree of importance of the specialist. Determination of the expert's degree of importance is done by means of an instrument of data collection.

d. Choice of linguistic terms and pertinence functions for the evaluation of predictive indicators: From the perspective of fuzzy theory, each predictive indicator can be seen as a linguistic variable, related to a set of linguistic terms associated to pertinence functions, in a previously established reference set. Each indicator will 
be a composition of linguistic terms, obtained in an evaluation process, made through the judgment of specialists. Therefore, they will also be fuzzy numbers.

e. Determination of the degree of importance of each predictive indicator. This step aims to obtain from the experts the degrees of importance of each of the indicators of each principle of resilience engineering, through the use of the set of linguistic terms, characterized by the triangular fuzzy numbers shown in Table 1

\subsection{Treatment of the Data}

The treatment of the data collected from the specialists in the evaluation of each predictive indicator is made using the similarity aggregation method proposed by HSU and CHEN (1996), a combination of the individual expert judgments are made. This step involves:

1) the calculation of the degree of agreement between opinions;

2) the construction of the concordance matrix;

3) calculation of relative agreement;

4) calculation of the relative degree of agreement;

5) the calculation of the consensus coefficient of the specialists;

6) the determination of the fuzzy value of each indicator referring to the principles of resilience engineering.

\section{The Context for Using Fuzzy Logic}

As already mentioned, the case to be analyzed with the use of Fuzzy logic is based on the inspection plan of an oil platform, more specifically an FPSO (Floating Production, Storage, and Offloading) that are floating units that have large tanks capable of storing high volumes of produced oil. In order to make clear the complexity of an oil platform with this, we can say that we have a large ship with a refinery mounted on top. Obviously, this is a simplification of what is an FPSO, but on the other hand, we can already have an idea of the number of pipes and equipment that we must have to manage. (Figure 5)

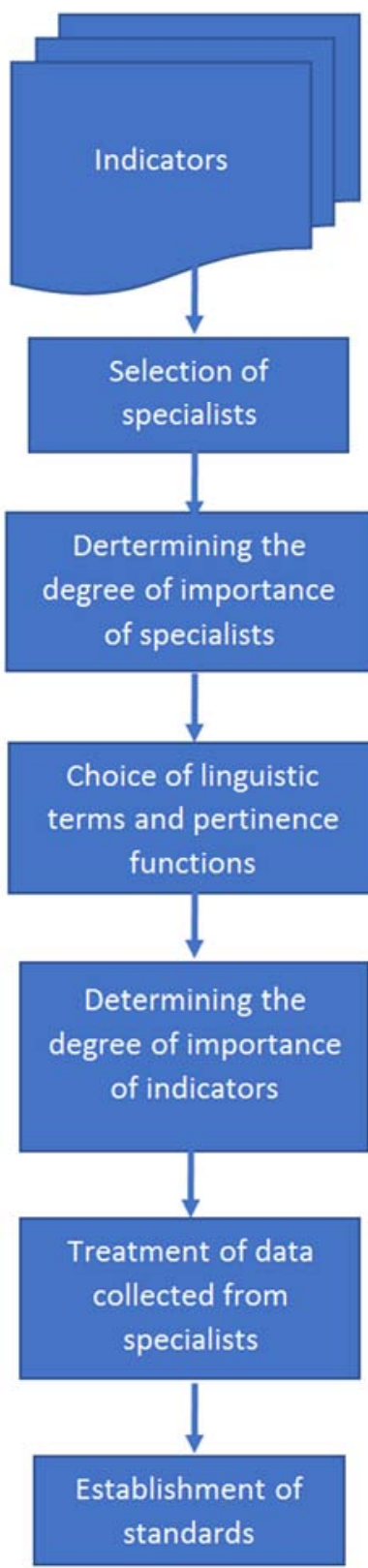

Figure 4. Step by step fuzzy methodology.

Table 1. Fuzzy numbers for linguistic terms.

\begin{tabular}{llll}
\hline Degree of importance & Symbology & Linguistic Term & Triangular fuzzy number \\
\hline O,0 & L & LOW & N1= $(0,0 ; 0,0 ; 1,0)$ \\
1,0 & M & MEDIUM & N2=(0,0;1,0;2,0) \\
2,0 & MH & MEDIUM HIGH & N3= (1,0;2,0;3,0) \\
3,0 & $\mathrm{H}$ & HIGH & $\mathrm{N} 4=(2,0 ; 3,0 ; 3,0)$ \\
\hline
\end{tabular}

In the inspection planning process, the severity of the consequences of failure shall determine the margin of safety to be applied to the periodicity implied by the RBI, taking account of the uncertainties in the corrosion rate prediction.

The predicted failure mechanisms shall be determined from the RBI and the most effective detection methods identified. Corrosion loops shall be identified and grouped which have similar corrosion reaction sensitivity $[8,11]$. The inspection efforts shall be focused on areas of the plant that have the highest corrosion risk and which are critical in terms of safety. Based on what has just been described, we can understand that there are certain key points in this process that have a high degree of uncertainty, such as the question of the damage mechanism. Imagine a new plant where there has never been an inspection and it is not known what will happen to the material of the tube or equipment due to the interactions of pressure, temperature, fluid, pipe geometry, material, number of curves, etc, etc. that means we do not have some kind of track record to feed the RBI system and get predictions about what to do to increase the life of the 
material. Usually, there is a corrosion engineer who has the responsibility to provide the RBI engineer with information on the mechanism of corrosion and degradation.

It is at this moment that we enter with the fuzzy logic, to analyze if it is possible to rank the main modes of corrosion damage, helping the engineers to make initial assumptions with less subjectivity.

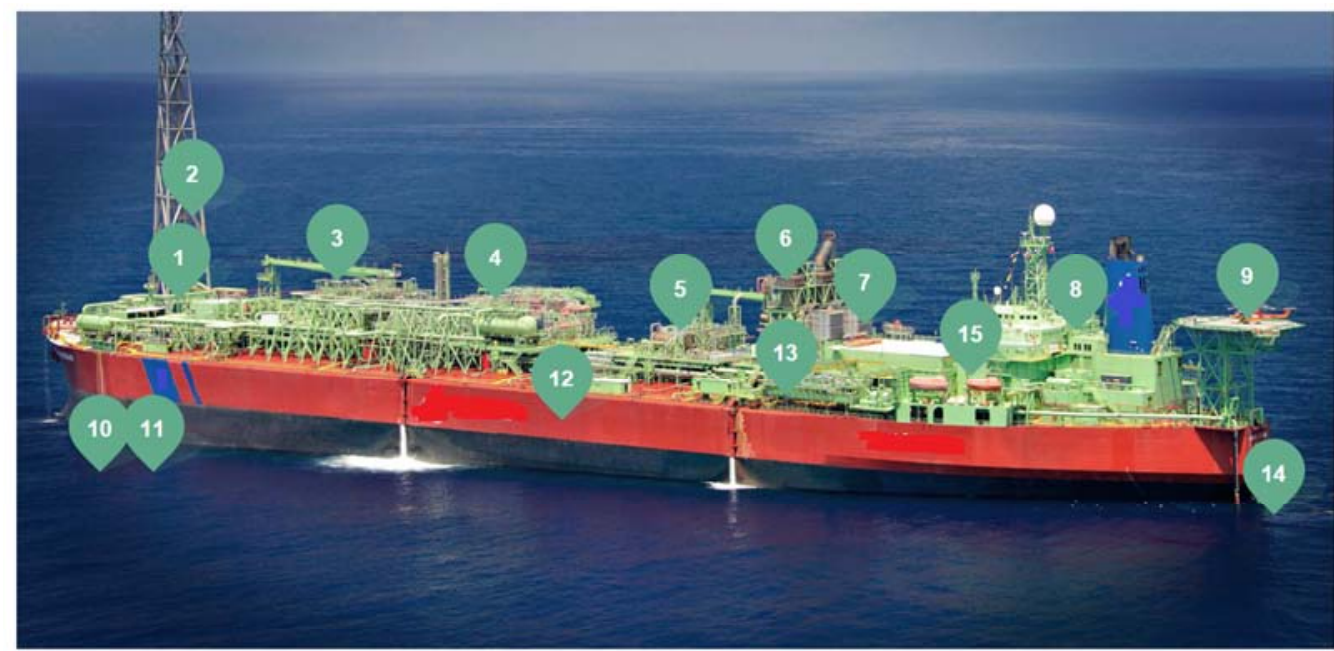

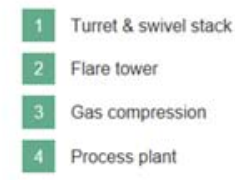

Process plant

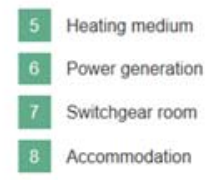

Figure 5. Main areas of the FPSO.

\section{Case Study}

Following the steps already mentioned in item 5.1, we must start by choosing the indicators, the parameters that affect the reduction of material thickness and consequently interfere with the inspection plan (programming).

\subsection{Indicators}

The indicators will be the mechanisms of corrosion damage. As already indicated in figure 2, the API gives us a list of the main mechanisms, however, we will use only 7 of these mechanisms, based on DNV RP G101 Appendix A [9].

1. $\mathrm{CO}_{2}$ corrosion (local/uniform)

2. External corrosion - atmospheric

3. Sand erosion

4. MIC - Microbiologically influenced corrosion

5. External SCC (chloride)

6. Flue gas dew point corrosion

7. Water corrosion

\subsection{Selection of Experts}

The fuzzy set theory allows representing elicited knowledge through pertinence functions. A fuzzy model built with the help of experts increases its accuracy as the number of specialists recognized by their knowledge, experience and work in the area of interest increases (ISHIKAWA et al., 1993) [10]. In this way, the selection of the specialists is a critical factor, since the reliability and quality of results depend on the quality of the specialists. The selection of the group of experts was carried out by means of a questionnaire. The idea was to use professionals who are involved both in the elaboration of the inspection plan and in the professionals, who execute and/or audit the inspection actions, such as:

a. E1 - Offshore Installation Manager

b. E2 - Technical Maintenance Manager

c. E3 - Integrity Engineer

d. E4 - HSE Manager

\subsection{Determination of the Degree of Importance of the Specialists}

Since a specialist group is usually heterogeneous, opinions cannot be considered with the same degree of importance. The determination of the degree of importance of the specialist is done by means of a data collection instrument. This instrument used for data collection is a questionnaire that was used by BELCHIOR (1997) and MORÉ (2004) to identify the profile of the specialist. Each questionnaire contains information from a GIE single specialist. The respective degrees of importance is defined as a subset $\mu \mathrm{i}(\mathrm{k})$ $\epsilon[0,1]$. The degree of importance of each specialist, GIEi, which is their relative degree of importance compared to other specialists, is defined by:

$$
G I E_{I}=\frac{t Q_{i}}{\sum_{i=1}^{n} t Q_{i}}
$$

Where:

$t Q_{i}=$ Total of points of the questionnaire for the specialist $\mathrm{i}$. 
$n=$ Number of questions

Table 2 shows the result.

As seen in Table 2 the specialist 4 has the highest degree of importance, followed by the specialist 2. Figure 6 Shows the comparative result among the specialists

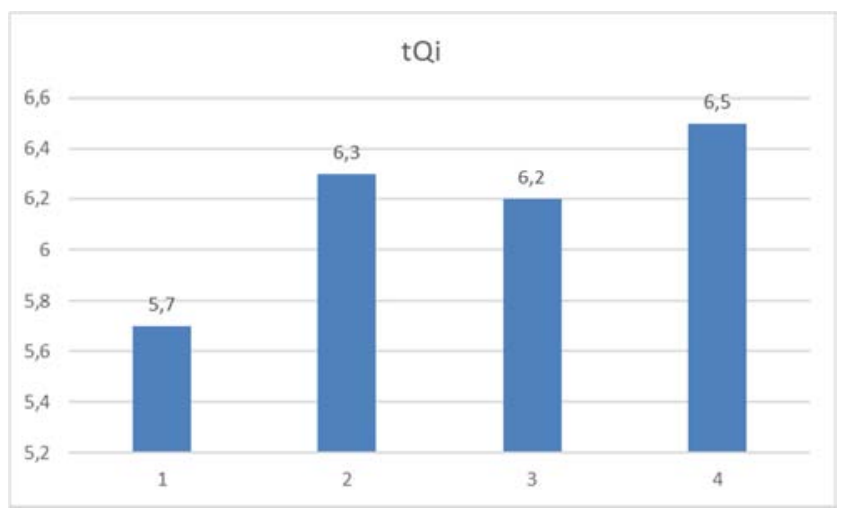

Figure 6. Graphical representation of the degrees of importance of specialists.

\subsection{Choice of Language Terms and Pertinence Functions}

From the perspective of fuzzy theory, each predictive indicator can be seen as a linguistic variable, related to a set of linguistic terms associated with pertinence functions, in a previously established reference set. Each indicator will be a composition of linguistic terms, obtained in an evaluation process, made through the judgment of specialists. Therefore, they will also be fuzzy numbers. The linguistic terms were defined as:

a. High $(\mathrm{H})$ : for indicators that are considered very important (have great influence);

b. Medium-High (MH): for indicators that are considered important (have influence);

c. Medium (M): for indicators that are considered to be of minor importance (have little influence);

d. Low (L): for indicators that are not considered important /have no influence.

These linguistic terms will be represented by triangular fuzzy numbers, which will denote the degree of importance of each indicator considered. According to PEDRYCZ (1994), the triangular fuzzy numbers deal very well with information with a high degree of uncertainty and uncertainty, as are the linguistic variables that translate the opinions of experts. Figure 7 shows the graph of their relevance functions.

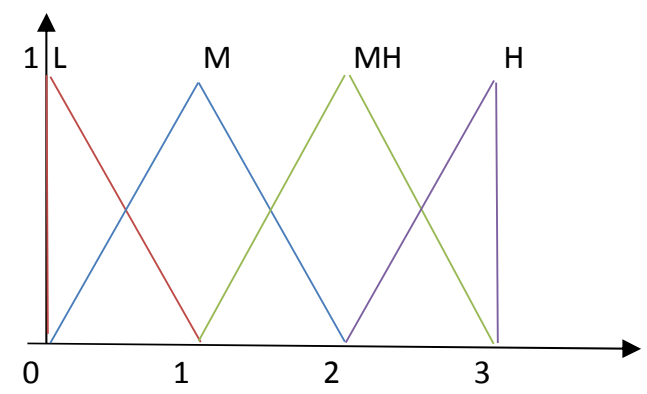

Figure 7. Graphical of relevance functions.
Table 2. Results of the degrees of importance of the experts Ei.

\begin{tabular}{llllllllllll}
\hline $\mathbf{E i}$ & $\mathbf{q 1}$ & $\mathbf{q 2}$ & $\mathbf{q 3}$ & $\mathbf{q 4}$ & $\mathbf{q 5}$ & $\mathbf{q 6}$ & $\mathbf{q 7}$ & $\mathbf{q 8}$ & $\mathbf{q 9}$ & $\mathbf{t Q i}$ & $\mathbf{G I E i}$ \\
\hline 1 & 1 & 0,4 & 0 & 0 & 0,7 & 0,7 & 1 & 0,9 & 1 & 5,7 & 0,231 \\
2 & 1 & 0,6 & 0 & 0 & 1 & 0,7 & 1 & 1 & 1 & 6,3 & 0,255 \\
3 & 1 & 0,6 & 0 & 0 & 1 & 0,7 & 1 & 0,9 & 1 & 6,2 & 0,251 \\
4 & 0,8 & 0,8 & 0,5 & 0,4 & 0,7 & 0,7 & 1 & 0,7 & 0,9 & 6,5 & 0,263 \\
& & & & & & & & & & 24,7 & 1 \\
\hline
\end{tabular}

\subsection{Determination of the Degree of Importance of Each Indicator}

The objective of this stage is to obtain from the experts the degrees of importance of each of the indicators, through the use of the set of linguistic terms, characterized by the triangular fuzzy numbers shown in Table 1. To determine the degree of importance of the indicators, a spreadsheet was used. This spreadsheet was filled by the experts, using the set of linguistic terms, characterized by the triangular fuzzy numbers shown in Table 1. The quality of the research depends on the level of transparency of the opinions provided by the experts. Fuzzy math will be used to aggregate these opinions and to elaborate a hierarchy of indicator.

\subsection{Treatment of Data Collected from Experts in the Evaluation of Each Indicator}

In this topic, we will begin the treatment of the data from the research with the 04 experts to evaluate the degrees of importance of the 7 indicators. The following steps detail the application of this method:

1) check the union and intersection areas of fuzzy opinions;

2) calculate the degree of agreement of opinions;

3 ) construct the concordance matrix;

4) calculate the relative concordance;

Table 3. Results of the degrees of importance of each indicator.

\begin{tabular}{lllll}
\hline Indicator & E1 & E2 & E3 & E4 \\
\hline sand erosion & L & L & L & L \\
$\mathrm{CO}_{2}$ corrosion (local/uniform) & $\mathrm{H}$ & $\mathrm{MH}$ & $\mathrm{MH}$ & $\mathrm{H}$ \\
MIC - Microbiologically influenced corrosion & $\mathrm{M}$ & $\mathrm{L}$ & $\mathrm{M}$ & $\mathrm{M}$ \\
External SCC (chloride) & $\mathrm{L}$ & $\mathrm{M}$ & $\mathrm{M}$ & $\mathrm{L}$ \\
external corrosion - atmospheric & $\mathrm{MH}$ & $\mathrm{M}$ & $\mathrm{M}$ & $\mathrm{MH}$ \\
flue gas dew point corrosion & $\mathrm{M}$ & $\mathrm{M}$ & $\mathrm{M}$ & $\mathrm{H}$ \\
water corrosion & $\mathrm{H}$ & $\mathrm{M}$ & $\mathrm{M}$ & $\mathrm{MH}$ \\
\hline
\end{tabular}

5) calculate the degree of relative concordance;

6) calculate the consensus coefficient of the specialists;

7) find the fuzzy value of each of the 7 chosen indicators.

As an example, in this step, the calculations of the evaluation of the indicator "external corrosion" are presented.

\subsubsection{Check the Union and Intersection Areas of Fuzzy Opinions}

In this item, we have to find the values of the areas of intersecting and union of Fuzzy opinions, which are shown in tables 4 and 5.

\subsubsection{Calculate the Degree of Agreement of Opinions}

The next step is to calculate the degree of agreement 
between the opinions (GC). This value is found by the ratio between the intersection area $(\mathrm{AI})$ and the union area (AU).

$$
\mathrm{GCij}=\frac{\mathrm{Ai}}{\mathrm{Au}}
$$

\subsubsection{Concordance Matrix}

The next step is to build the concordance matrix (MC), which is obtained by combining all degrees of agreement between each pair of experts. See table 6 .

As we have selected four experts, the concordance matrix will be a symmetric matrix $(\mathrm{GCij}=\mathrm{GCji})$ of 4 rows and 4 columns, with all elements of its diagonal equal to 1

Table 4. Values of the intersecting area.

\begin{tabular}{lllll}
\hline Opinions & MH & M & M & MH \\
\hline MH & 1 & 0,25 & 0,25 & 1 \\
M & 0,25 & 1 & 1 & 0,25 \\
M & 0,25 & 1 & 1 & 0,25 \\
MH & 1 & 0,25 & 0,25 & 1 \\
\hline
\end{tabular}

Table 5. Values of a union area.

\begin{tabular}{lllll}
\hline Opinions & MH & M & M & MH \\
\hline MH & 1 & 1,75 & 1,75 & 1 \\
M & 1,75 & 1 & 1 & 1,75 \\
M & 1,75 & 1 & 1 & 1,75 \\
MH & 1 & 1,75 & 1,75 & 1 \\
\hline
\end{tabular}

Table 6. Concordance matrix.

\begin{tabular}{llll}
\hline $\mathbf{1}$ & $\mathbf{0 , 1 4 2 8 5 7}$ & $\mathbf{0 , 1 4 2 8 5 7}$ & $\mathbf{1}$ \\
\hline 0,14285714 & 1 & 1 & 0,142857 \\
0,14285714 & 1 & 1 & 0,142857 \\
1 & 0,142857 & 0,142857 & 1 \\
\hline
\end{tabular}

\subsubsection{Calculate the Relative Concordance}

In this topic we will calculate the relative concordance $\left(\mathrm{Cr}_{\mathrm{i}}\right)$ of each specialist, based on the following formula:

$$
\mathrm{CR}_{\mathrm{i}}=\operatorname{SQRT}\left((1 / \mathrm{n}-1) \Sigma_{\mathrm{j}=1}^{\mathrm{n}}(\mathrm{GCij})^{2}\right)
$$

\subsubsection{Calculation of Relative Grade of Concordance}

The degree of relative agreement $\left(\mathrm{GCR}_{\mathrm{k}}\right)$ of each specialist, in relation to the other specialists is obtained by the equation below:

$$
\mathrm{GCC}_{K}=\frac{C R_{i}}{\sum n_{k=1} C R k}
$$

Table 7. Relative concordance.

\begin{tabular}{ll}
\hline Experts & $\mathbf{C R}_{\mathbf{i}}$ \\
\hline 1 & 0,824786 \\
2 & 0,824786 \\
3 & 0,824786 \\
4 & 0,824786 \\
Total & 3,299144 \\
\hline
\end{tabular}

Table 8. Relative grade of concordance.

\begin{tabular}{ll}
\hline Experts & $\mathbf{G C R}_{\mathbf{k}}$ \\
\hline 1 & 0,25 \\
2 & 0,25 \\
3 & 0,25 \\
4 & 0,25 \\
\hline
\end{tabular}

\subsubsection{Calculation the Consensus Coefficient of the Specialists}

The coefficient of consensus of each speciesist (CCEk), is calculated according to the formula below:

$$
C C E_{K}=\frac{G C R_{K^{*}} G I E i}{\sum_{i=0}^{n} G C R_{K^{*}} G I E i}
$$

\subsubsection{Fuzzy Value}

After obtaining the consensus coefficient of the 4 specialists, we will have the last step, which consists in the calculation of the Fuzzy value of each of the chosen indicators.

$$
\mathrm{N}=\Sigma_{\mathrm{i}=1}^{\mathrm{n}}\left(\mathrm{CCEi}^{*} \text { ni }\right)
$$

where $n_{i}$ is the triangular Fuzzy number relative to the linguistic terms used by the experts in the evaluation of the indicators. Table 10 shows the Fuzzy numbers found for each chosen index.

\begin{tabular}{ll} 
& Table 9. Coefficient of consensus. \\
\hline Experts & CCEk \\
\hline 1 & 0,230769 \\
2 & 0,255061 \\
3 & 0,251012 \\
4 & 0,263158 \\
\hline
\end{tabular}

\begin{tabular}{lllll}
\multicolumn{5}{c}{ Table 10. Fuzzy Numbers. } \\
\hline \multirow{2}{*}{ Indicators } & \multicolumn{4}{l}{ Fuzzy Numbers } \\
\cline { 2 - 5 } & $\boldsymbol{a}$ & $\boldsymbol{b}$ & $\mathbf{c}$ & GII \\
\hline $\mathrm{CO}_{2}$ corrosion & 1,493927 & 2,493927 & 3 & 1 \\
External corrosion & 0,493927 & 1,493927 & 2,493927 & 0,599026 \\
Sand erosion & 0 & 0 & 1 & 0 \\
MIC & 0 & 0,827935 & 1,827935 & 0,331981 \\
External SCC & 0 & 0,506073 & 1,506073 & 0,202922 \\
flue gas & 0 & 0,506073 & 1,506073 & 0,202922 \\
Water corrosion & 0,609275 & 1,609275 & 2,419288 & 0,645277 \\
\hline
\end{tabular}

\section{Results and Discussions}

After we have found the Fuzzy numbers for each indicator, we now need to prioritize the obtained values, and to do so it is necessary to calculate the degree of relevance of each of these. The degree of relevance of each indicator (GRFi) is obtained by normalizing the values of these indicators. Such normalization is performed using the following expression:

$$
G R F_{i}=\frac{b_{i}}{\operatorname{Major}(b)}
$$

In the above equation, major (b) is the largest value of $b$ of the triangular fuzzy number found for each indicator. We choose $b_{i}$ because this value corresponds to a degree of membership of 1 . Therefore, GRI will be the crisp value of each of the indicators. The analysis of the results obtained in Table 10 indicates that the highest value of $b$ occurs in the $\mathrm{CO}_{2}$ corrosion indicator.

Thus, it has to be greater $(b)=2.49$, leading to a degree of relevance equal to 1 for this indicator, followed by the water corrosion indicator, as can be seen in table 11, which shows 
the hierarchy in descending order of the seven indicators.

Table 11. Values in descending order.

\begin{tabular}{ll}
\hline Indicators & GII \\
\hline $\mathrm{CO}_{2}$ Corrosion & 1 \\
Water corrosion & 0,645277 \\
External corrosion & 0,599026 \\
$\mathrm{MIC}$ & 0,331981 \\
external SCC & 0,202922 \\
flue gas & 0,202922 \\
Sand erosion & 0 \\
\hline
\end{tabular}

\section{Conclusions}

In this study, we present the concept of Fuzzy logic, applied to an inspection plan of an FPSO. This methodology allows listing a ranking of the mechanisms of damage most impacting to the materials of pipes and equipment. This information is very important if we want to manage the risks of a process plant that is located in a floating unit, producing and storing oil. The result shown in table 11 shows that the 3 major damage mechanisms are: $\mathrm{CO}_{2}$ corrosion, water corrosion, and external corrosion. We can now compare this result with the results of the inspections carried out in the process area. If we look at the rates of wall reduction due to corrosion ( $\mathrm{mm} /$ year), we can see that the highest rates were caused by corrosion by water and corrosion by $\mathrm{CO}_{2}$. Water corrosion appears more than pro- $\mathrm{CO}_{2}$ corrosion by having more equipment and piping where the water participates more in the process. However, it is clear that the first two mechanisms of damage pointed out by Fuzzy logic are the ones that caused more damage, more reduction on the walls of pipes and equipment. Now, with this result in hand, the team responsible for determining which equipment and tubes will be inspected and at what intervals, gains more objectivity at the time of planning, since it knows exactly which mechanisms should be prioritized, which means that there has been an increased reliability of the inspection, a reduction in the likelihood of accidents and an increase in the level of process safety.

\section{References}

[1] Khan, Faisal I, Sadiq, Rehan, Husain, Tahir. Risk-based process safety assessment and control measures design for offshore process facilities. Available at https://doi.org/10.1016/S0304-3894 (02)00004-3

[2] Damage Mechanisms Affecting Fixed Equipment in the Refining Industry. API RECOMMENDED PRACTICE 571 SECOND EDITION, APRIL 2011 (page 4-65).
[3] Abordagem Sobre Inspeção Baseada Em Risco Segundo Api581. Daniel K; Mishina V. II National Congress of Mechanical Engineering; 2012.

[4] BW Offshore - Risk Based Inspection for Pressure Static Equipment Doc. no: MS-MP01650 Rev: 5 Published: 07.01.2019 (Page 4).

[5] Markowski, Adam S. Mannan, M. Sam Fuzzy risk matrix. Available at https://doi.org10.1016/j.jhazmat.2008.03.055

[6] Grecco, Cláudio Henrique dos Santos Avaliação da resiliência em organizações que lidam com tecnologias perigosas: o caso da expedição de radiofármacos / Cláudio Henrique dos Santos Grecco. - Rio de Janeiro: UFRJ/COPPE, 2012. XIV, 154 p.: il.; p. 131-140.

[7] Vianna Filho, Alfredo Marques Análise da disponibilidade da instrumentação nuclear de um reator de pesquisa / Alfredo Marques Vianna Filho. - Rio de Janeiro: UFRJ/COPPE, 2016. XI, 133 p.: il.;. Referências Bibliográficas: p. 111-116.

[8] BW Offshore - Corrosion Management Strategy Doc. no: MSMP01638 Rev:1 Issue:7/3/2015 (Page 14).

[9] Recommended Practice DNV-RP-G101 Risk Based Inspection of Offshore Topsides STATIC Mechanical Equipment October 2010 - Appendix A (Page 22).

[10] Santos, Luana Fernanda Marques. Indicadores proativos de segurança de processo em instalações de armazenamento e transporte dutoviário de petróleo e derivados: construção de um painel de indicadores/ Luana Fernanda Marques Santos 2018. 320 f.: il.:38 f. 175-184.

[11] BW Offshore. Corrosion Management Doc. no: MSMP01637. Rev:1 Issue:7/3/2015.

[12] Chi-Hui Chien, Chun-Hung Chen, Yuh J. Chao. A strategy for the risk-based inspection of pressure safety valves. DOI: $10.1016 /$ j.ress.2008.09.002

[13] FERNANDES, Simone Zappe Análise da segurança de processo baseada em riscos no ciclo de vida das plataformas de petróleo./ Simone Zappe Fernandes. - 2018. f: 147 p il. $30 \mathrm{~cm}$.

[14] Grecco, Cláudio Henrique dos Santos Avaliação da resiliência em organizações que lidam com tecnologias perigosas: o caso da expedição de radiofármacos / Cláudio Henrique dos Santos Grecco. - Rio de Janeiro: UFRJ/COPPE, 2012. XIV, 154 p.: il.; $29,7 \mathrm{~cm}$.

[15] Luiz Antonio Moschini de Souza. Inspeção de sistemas de tubulações- Rio de Janeiro: IBP, 2017. 68 p.; il. (Guia de Inspeção, n. 06). E-ISBN 978-85-9486-083-5

[16] Maneesh Singh, Marshal Pokhrel. A Fuzzy logic-possibilistic methodology for risk-based inspection (RBI) planning of oil and gas piping subjected to microbiologically influenced corrosion (MIC). DOI: 10.1016/j.ijpvp.2017.11.005 